\title{
Scirrhous Hepatocellular Carcinoma: Systematic Review and Pooled Data Analysis of Clinical, Radiological, and Histopathological Features
}

\author{
Anastasia Murtha- \\ Lemekhova (D) ${ }^{1,2}$ \\ Juri Fuchs id 1,2 \\ Erik Schulz ${ }^{1,2}$ \\ Anthe Suzan Sterkenburg' \\ Philipp Mayer iD ${ }^{2,3}$ \\ Jan Pfeiffenberger ${ }^{2,4}$ \\ Katrin Hoffmann ${ }^{1,2}$ \\ 'Department of General, Visceral, and \\ Transplantation Surgery, Heidelberg \\ University Hospital, Heidelberg, \\ Germany; ${ }^{2}$ RELIVE Initiative, Heidelberg \\ University Hospital, Heidelberg, \\ Germany; ${ }^{3}$ Department of Diagnostic and \\ Interventional Radiology, Heidelberg \\ University Hospital, Heidelberg, \\ Germany; ${ }^{4}$ Department of \\ Gastroenterology and Hepatology, \\ Heidelberg University Hospital, \\ Heidelberg, Germany
}

Correspondence: Katrin Hoffmann Department of General, Visceral, and Transplantation Surgery, Heidelberg University Hospital, Im Neuenheimer Feld 420, Heidelberg, 69120, Germany Tel +496221566110

Fax +496221565450 Email katrin.hoffmann@med.uni-heidelberg. de
Background: Aberrant subtypes of hepatocellular carcinoma (HCC) account for $20-30 \%$ of all HCCs and habitually present a challenge in diagnosis and treatment. Scirrhous hepatocellular carcinoma (s-HCC) is often misdiagnosed as cholangiocarcinoma, fibrolamellar hepatocellular carcinoma, or metastasis.

Methods: Electronic databases (PubMed, Web of Knowledge, Google Scholar, Cochrane Library, and WHO International Clinical Trials Registry Platform) were searched for publications on scirrhous hepatocellular carcinoma without date or language restrictions. Quality assessment was performed using a tool proposed by Murad et al for case reports and series. For observational studies, MINORS quality assessment tool was used. This study was registered at PROSPERO (CRD42020212323).

Results: S-HCC arises in patients with chronic hepatitis (hepatitis B in $60 \%$ and hepatitis $\mathrm{C}$ in $21 \%$ ). S-HCC primarily affects men with a mean age of 55.8 years. Serum AFP is elevated above $20 \mathrm{IU} / \mathrm{mL}$ in $66.7 \%$ of the patients. On ultrasound, s-HCC presents as hypoechoic or mosaic pattern lesions $(47.6 \%$ each) and causes a retraction of the liver surface $(70 \%)$ when near the capsule. Delayed enhancement of the tumor is evident in $87.0 \%$. On MRI, $65.0 \%$ of s-HCCs show a target appearance. Histopathologic pattern is mostly irregular (97.6\%). Lesions show a bulging appearance $(100 \%)$, septae $(85.6 \%)$ and a central scar $(63.5 \%)$, and usually lack central necrosis $(75 \%)$. Immunohistochemistry shows HepPar 1 positivity in $64.6 \%$, CK7 in 40.7\%, and EMA in $41.9 \%$. The 5-year overall survival rate estimates $45.2 \%$ and $45.5 \%$ of the patients experience a recurrence after hepatectomy.

Conclusion: S-HCC is a rare subtype of HCC primarily arising in hepatitis- or cirrhosisafflicted livers and incorporates atypical radiological and histopathological HCC features. Despite lower recurrence rates, overall survival of patients with s-HCC is poorer than generally for HCC, underlining the need for individualized treatment. Patients with atypical lesions of the liver should be referred to tertiary hospitals for interdisciplinary assessment and treatment.

Keywords: scirrhous HCC, s-HCC, rare liver tumors, RELIVE initiative

\section{Introduction}

Scirrhous hepatocellular carcinoma (s-HCC) is a rare morphologic subtype of hepatocellular carcinoma accounting for an estimated $4 \%$ of all HCCs. Only recently has it been recognized as a subtype of HCC in the WHO classification. ${ }^{1}$ HCC poses high morbidity and mortality risks, becoming the fourth most common cause of cancerrelated mortality. ${ }^{2}$ Atypical HCCs comprise about $20-30 \%$ of all HCC cases; however, this estimation is based on limited data. ${ }^{3}$ Further analysis in order to estimate the 
impact of morphological subtypes is needed to steer $\mathrm{HCC}$ treatment forward, towards individual cancer therapy.

$\mathrm{S}-\mathrm{HCC}$ is often misdiagnosed radiologically as cholangiocarcinoma, fibrolamellar hepatocellular carcinoma (FLHCC), or even metastasis. ${ }^{4-7}$ Yet, for adequate treatment, correct diagnosis is indispensable. Although morphologically there are some similarities between scirrhous and fibrolamellar HCCs, only an adequate histological work-up can lead to the correct diagnosis. S-HCC shows abundant fibrous stroma, which accounts for $30-50 \%$ of the tumor mass, concentrated at the center, with nests of densely associated tumor cells. ${ }^{3}$ While FL-HCC occurs primarily in young patients without liver disease, ${ }^{8}$ s-HCC tends to affect patients with chronic hepatitis or cirrhosis. A crucial aspect of diagnosis is molecular testing, as DNAJB1-PRKACA oncogenic fusion is a sensitive marker for FL-HCC, albeit potentially non-specific. ${ }^{9}$ Detailed future clinical characterization of patients may be revealing for the differentiation between s-HCC and other lesions.

It is unclear, how the outcomes of s-HCC compare to the conventional subtype and therefore patients receive the same recommendations despite striking differences in the pathology of the lesions and associated comorbidities. Comparative studies on chemotherapy response in different subtypes of HCCs are also lacking. Comprehensive molecular analysis of each subtype of HCC is necessary to achieve a satisfactory level of expertise and with it structure the targeted approach for adequate patient-oriented treatment. International clinical registries for atypical HCCs could close the informational gap and generate data for an evidence-based approach. The aim of this analysis is to examine available data on clinical, radiological, and histopathological features of s-HCC to summarize the currently available knowledge and guide further research into HCC subtypes.

\section{Methods}

The systematic review is reported in accordance with the current PRISMA guidelines. ${ }^{10}$ The review methodology was established before commencement. The protocol of the study was registered prior to data extraction on PROSPERO platform (CRD42020212323). ${ }^{11}$

\section{Literature Search}

A literature search was systematically conducted according to the recent recommendations of the Cochrane Collaboration. $^{12}$ The aim of the search was to identify all reports on scirrhous hepatocellular carcinoma. The searches were performed using databases MEDLINE via PubMed, Web of Knowledge, Google Scholar, Cochrane Library, and WHO International Clinical Trials Registry Platform. An additional hand search was completed using the references of included studies. The last search was performed on February 12, 2021. No language or data restriction was implemented. The details of the search strategy for MEDLINE are provided in the Supplementary Materials.

\section{Study Selection}

No restriction was applied to the study design of the publications as a scarcity of studies on s-HCC was anticipated. All studies describing histological, radiological, or clinical features of s-HCCs were included. Publications such as comments, editorials, meeting abstracts, correspondence, and reviews were excluded. The screening of titles, abstracts, as well as of full texts was carried out by two independent reviewers (AML and JF). All disagreements were resolved by consensus.

\section{Data Extraction}

Data extraction of included studies was performed independently by two reviewers (AML and JF) using a standardized form prepared beforehand. Following data were extracted for each study: title of the publication, year, authors, country, journal, source of funding, study design, number in the cohort, patient characteristics, intervention, radiological description of the tumors, histological classifications and properties of the tumors, and clinical outcomes, including follow-up and recurrence rates.

\section{Statistical Analysis}

Pooled data analysis of characteristics involved descriptive statistics and most results were presented as percentages. Continuous variables were presented as means with standard deviation. Statistical analysis was performed using $\mathrm{R}$ version $4.0 .3 .^{13}$

\section{Results}

A total of 494 records were identified through databases and hand-searches. After exclusion of duplicated, 333 records were reviewed based on title and abstract by two independent reviewers. A total of 165 articles were further assessed by full-text review by two independent reviewers. Thirty-one articles were included in the qualitative synthesis and a total of 31 articles were included in various aspects of quantitative analyses. Figure 1 illustrates the study selection process. 

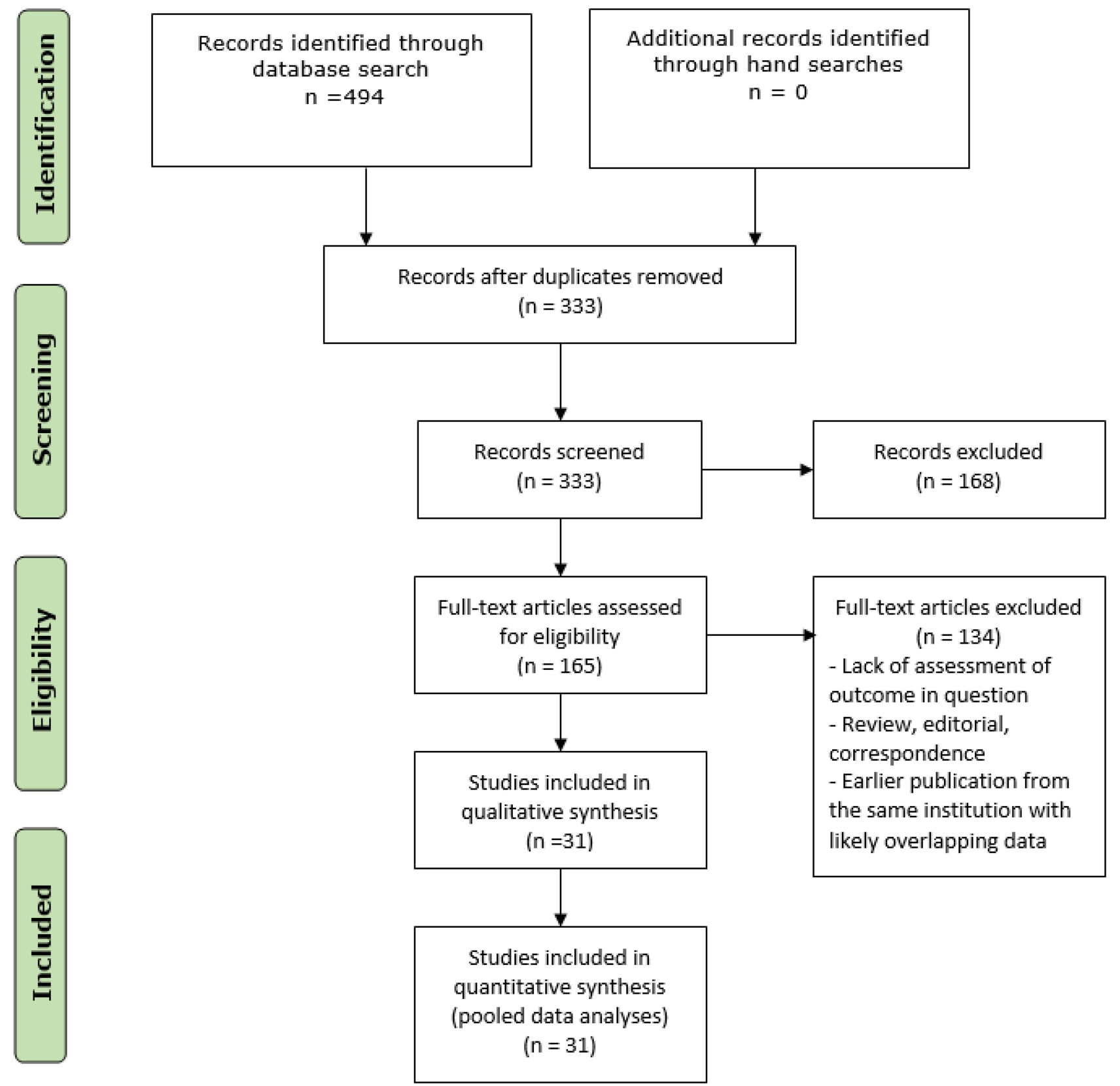

Diagram I Flow chart of the study selection process.

All included publications were of retrospective design with the majority being case reports and reports of series. $^{4,5,14-41}$ Due to scarcity of publications from the US and Taiwan, National Registry analyses of 161 and 30 patients, respectively, were included in the analysis. ${ }^{39,42}$

\section{Critical Appraisal of Included Studies}

The methodological quality assessment of all included studies was performed using a tool proposed by Murad et $\mathrm{al}^{43}$ due to the nature of the review, predominantly case reports and series, lack of a comparison group, and for comparability of studies. Detailed information about assessed qualities is provided as supplementary material (Supplementary Table 1). For observational studies, methodological index for non-randomized studies (MINORS) ${ }^{44}$ was additionally used to assess risk of bias (Supplementary Table 2).

\section{Demographics and Clinical Characteristics}

The distribution of available literature on s-HCC shows a certain predominance. Countries largely contributing 
articles on this subtype of HCC are Japan and South Korea (Figure 1).

The proportions are somewhat different in population sizes described (Figure 2). Although over $45 \%$ of all articles on s-HCC are published from Japanese hospitals, $25 \%$ of all patients identified in this review were treated in Japan. Only three publications originated from the US, the largest one, deriving its information from the National Registry, involved 161 patients, making it the secondlargest contributor in terms of population. ${ }^{39}$

Cumulatively, South Korea describes the largest population in their publications, with Japan being the third largest contributor.

Mean pooled population age was 55.8 years $( \pm 9.2$ years), ranging from 24 to 78 years. The pooled cohort consisted of 480 males and 154 females, equivalent to a male:female ratio of $3: 1$. Only five case reports $^{14,26,32,38,40}$ described initial clinical presentations: two patients had no symptoms and presented for hepatitis C follow-up, two patients experienced upper abdominal pain, and one presented with back pain due to metastatic disease. A total of 474 patients had preoperative information on the presence of an underlying liver disease: $59.7 \%$ (283 of 474 patients) had hepatitis B and 20.8\% (99 of 474 patients) had a history of hepatitis C. Information on $\mathrm{NASH}$ was provided in two publications - in a cumulative cohort of 61 patients, NASH was present in 10 cases. $^{31,37}$ Alcohol overconsumption was described for
10 patients in 7 publications reporting on a total of 139 patients, amounting to $7.2 \%$. AFP was provided in 15 publications. Serum AFP was increased above $20 \mathrm{IU} / \mathrm{mL}$ in $70.3 \%$ (26 patients) with maximum reaching $2372 \mathrm{IU} /$ $\mathrm{mL}$ in a pooled cohort of 37 patients (Table 1 ).

\section{Radiological Features}

In total, 14 articles reported radiological features of the analyzed s-HCCs. $5,14-18,21,23,25,27,33,37,38,41$ Most frequently reported prominent radiological features on ultrasound, CT, and MRI were selected and quantified (Table 2). On ultrasound, most s-HCC lesions presented as lesions with hypoechoic or mosaic patterns (each $47.6 \%$ ), while an isoechoic presentation was uncommon (4.8\%). For lesions described as located near the capsule of the liver, retraction of the liver surface was present in 70\% (14 of pooled 20 lesions). On CT scans, most lesions were well demarcated (66 of 122 cases [54.1\%]). Sixty-seven percent presented with lobulated contours (41 of 61 cases). Persistent hyperdensity of the tumor in the delayed phase was reported in $87.0 \%$ of the lesions ( 80 of 92 cases). On MRI, target appearance was described in $65.0 \%$ of the patients (97 of 149 cases) in the hepatobiliary phase.

\section{Histopathological Features}

Twenty-four articles $4,5,14-16,18-27,29-33,36,38,41,42$ provided details on histopathological features of the tumors, summarized in Table 3.

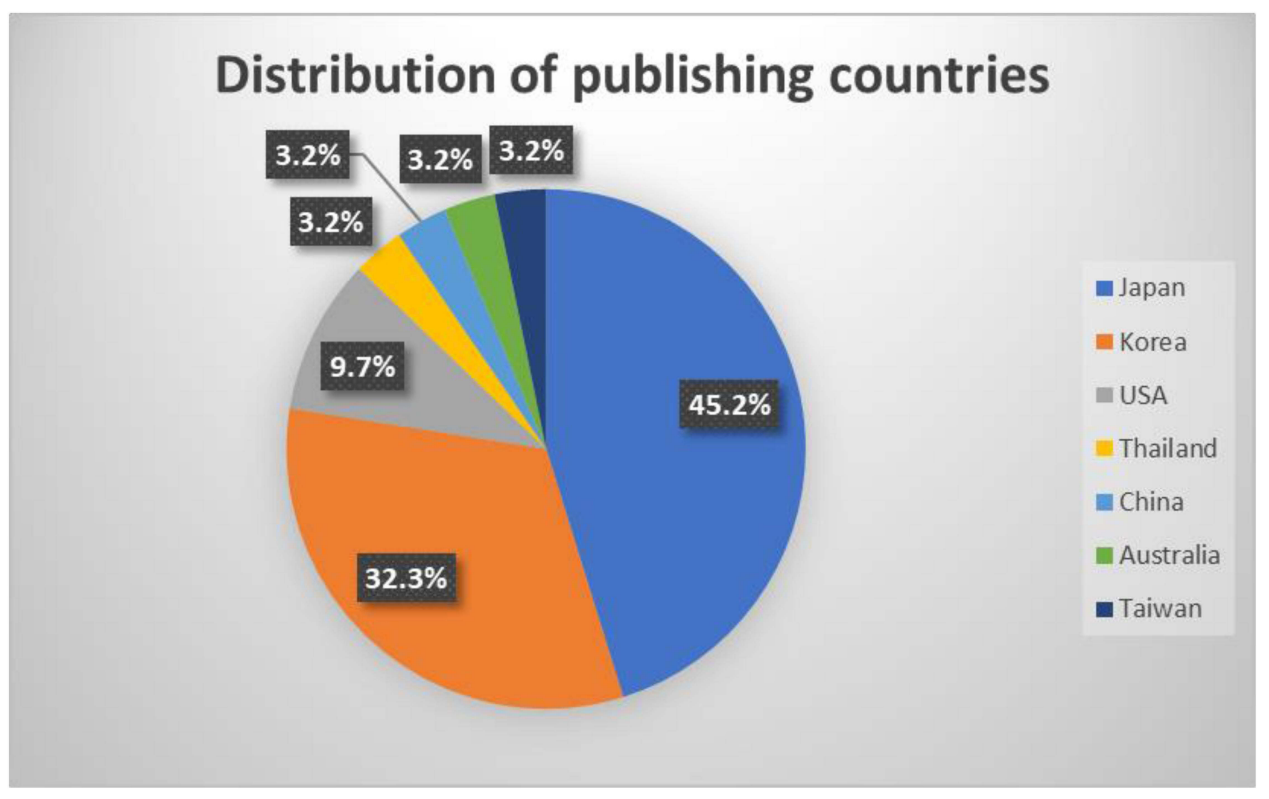

Figure I Distribution of countries to contribute publications on s-HCC. 


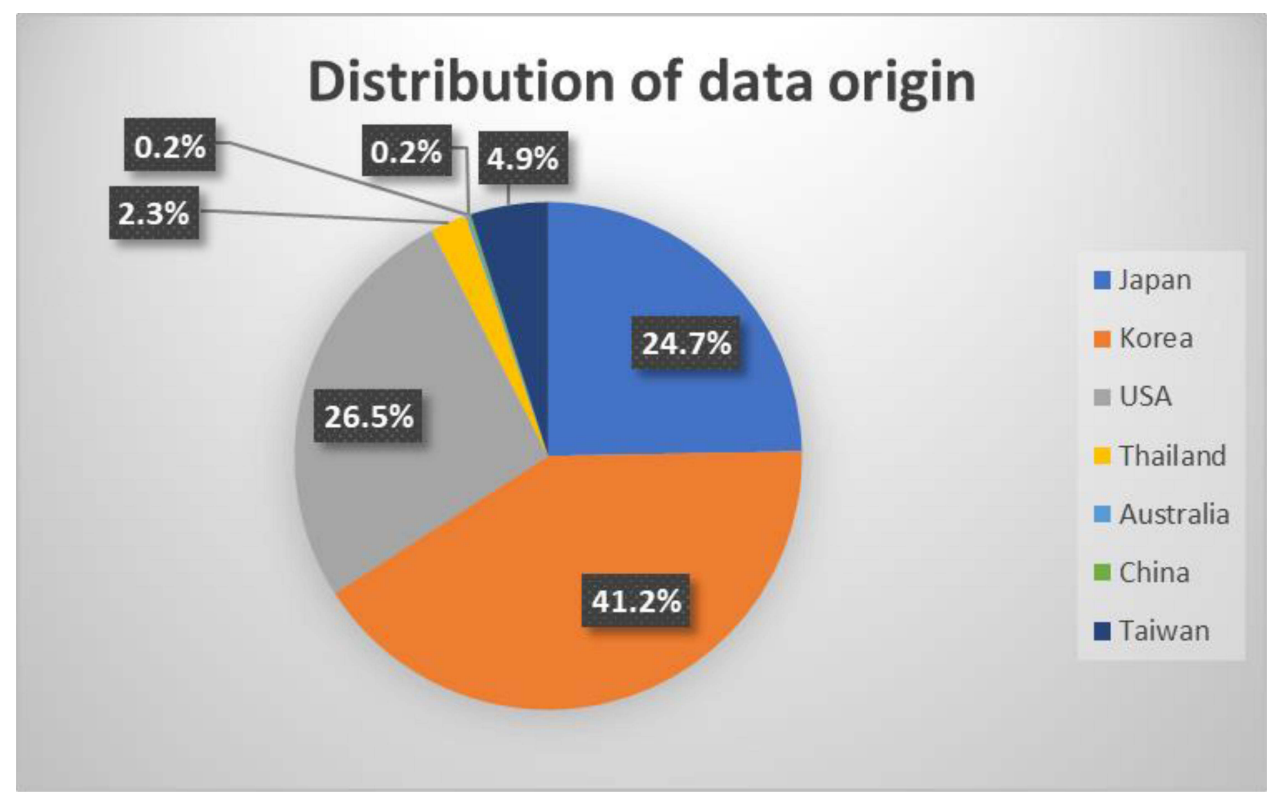

Figure 2 Distribution of origin of population data.

Of the patients, $82.9 \%$ had a solitary tumor (136 of 164 cases), while $17.1 \%$ presented with multiple lesions (28 of 164 cases). The tumor size ranged from 1.2 to $17.0 \mathrm{~cm}$ with a pooled mean and standard deviation estimated at $4.2 \pm 2.33 \mathrm{~cm}$. Edmondson-Steiner (ES) grade was provided in 228 cases. $77.6 \%$ had ES grade I/II while $22.4 \%$ had grade III/IV. More specific ES grading was provided in 133 cases: while $30.1 \%$ had grade I, $50.4 \%$ had grade II, $18.0 \%$ had grade III and $1.5 \%$ were diagnosed with grade IV. WHO tumor grading was defined in 227 cases: $25.5 \%$ had Grade I, while $50.7 \%$ and $23.8 \%$ had Grade II and III, respectively. Due to multiple revisions of the AJCC staging system, no synthesis of TNM staging could be performed for included studies.

Background liver parenchyma was described in 19 publications. Of 314 patients, $69.0 \%$ (216 of 313 cases) had either cirrhosis or chronic hepatitis in the rest of the liver. Hepatitis was more prevalent amounting to $39.9 \%$ of all cases (117 of 293 patients), while cirrhosis was evident in $28.3 \%$ ( 83 of 293 cases). Normal liver parenchyma was described in $11.8 \%$ of the patients (37 of 313 cases). Steatosis was assessed by only two publications, with a total of 57 patients: $63.2 \%$ (36 of 57 cases) had steatosis on histopathology.

In $97.6 \%$ of the cases ( 41 of 42 patients), an irregular histopathological structure of the border was described. For cases close to the capsule, bulging appearance was described in $100 \%$ of pooled cases (51 of 51). A complete capsule was described in $26.3 \%$ of the patients ( 82 of 312 cases), although an incomplete capsule was present in 43 more. A septum was present in $85.6 \%$ of the patients $(83$ of 97 cases), while a central scar was reported for $63.5 \%$ (40 of 63 cases). At the time of diagnosis/resection, venous invasion was present in $32.6 \%$ (46 of 141 cases). Most cases (174 of 232 cases) presented without necrosis, amounting to $75 \%$.

Only 14 studies $^{4,5,14,15,18,20-22,24,27,30,31,35,36}$ described immunohistochemical properties of the tumors summarized in Table 4. For targets analyzed in more than one publication, data is provided in Table 4. HepPar1 was evident in $64.6 \%$ of the patients (64 of 99 cases), while

Table I Demographics of Patients

\begin{tabular}{|l|l|}
\hline Feature & Metrics \\
\hline Age & \\
Mean \pm SD & $55.8 \pm 9.2$ years \\
Range & $24-78$ years \\
\hline Gender & \\
Male [n] & 480 \\
Female [n] & 154 \\
\hline Aetiology of liver disease & \\
Hepatitis B & $59.7 \%(283 / 474)$ \\
Hepatitis C & $20.8 \%(99 / 474)$ \\
NASH & $16.4 \%(10 / 61)$ \\
Alcohol & $7.2 \%(10 / 139)$ \\
\hline AFP & \\
$>20$ IU/mL & $70.3 \%(26 / 37)$ \\
\hline
\end{tabular}


Table 2 Radiological Features of s-HCCs

\begin{tabular}{|l|l|l|}
\hline Imaging Modality & Criterion & Prevalence \\
\hline Ultrasound & Pattern: & \\
& Hypoechoic & $47.6 \%(I 0 / 2 I)$ \\
& Mosaic & $47.6 \%(I 0 / 2 I)$ \\
& Isoechoic & $4.8 \%(I / 2 I)$ \\
\hline CT & Liver retraction & $70 \%(I 4 / 20)$ \\
& Well demarcated & $54.1 \%(66 / I 22)$ \\
& Lobulated contour & $67 \%(4 I / 6 I)$ \\
& Persistent & $87 \%(80 / 92)$ \\
& hyperdensity in the & \\
& delayed phase & \\
\hline MRI & Target appearance on & $65.0 \%(97 / I 49)$ \\
& HBP & \\
\hline
\end{tabular}

CK 7 was positive in $40.7 \%$ of the patients (35 of 86 cases). CK 19 was evident in 16\% (20 of 125 cases). EMA was positive in $41.9 \%$ of the patients (18 of 43 cases) (Table 4).

\section{Clinical Outcomes}

Fifteen publications ${ }^{14,24,27-31,36,37,39,42}$ provided information on follow-up. The follow-up ranged from 5 to 138 months. In $45.5 \%$ of the patients, a recurrence of s-HCC occurred during the follow-up period ( 70 of 154 cases) and $35.7 \%$ of the patients died of disease during the follow-up period (20 of 56 cases). A 5-year overall survival was reported between $21.1 \%$ and $73.3 \%$ with a pooled mean of $45.2 \%$. All patients with reported outcomes have undergone hepatectomy.

\section{Discussion}

Analysis of HCC morphological subtypes is essential to evolve treatment algorithms. With first-line treatment achieving a subpar median overall survival of merely 11-13 months ${ }^{45,46}$ and recurrence rates after hepatectomy remaining high, the development of new therapies based on specific pathophysiological characteristics and a personalized approach is the only strategy to ensure no HCC patient is left behind. As numerous scores have been unable to sufficiently identify patients at risk for HCC development, recurrence, or progression, $^{47}$ translational research remains the only approach with potential. After identifying fundamental clinicopathological features of specific subtypes of HCC, associated genetic and molecular signal transduction elements can then be studied to improve prognostic accuracy and adjust
Table 3 Histopathologic Features of s-HCCs

\begin{tabular}{|c|c|}
\hline Histologic Finding & Metrics \\
\hline Single tumor [\%] & $82.9 \%(136 / 164)$ \\
\hline Multiple tumors [\%] & $17.1 \%(28 / 164)$ \\
\hline \multicolumn{2}{|l|}{ Tumor size } \\
\hline Range & $1.2-17 \mathrm{~cm}$ \\
\hline Mean \pm SD & $4.2 \pm 2.33 \mathrm{~cm}$ \\
\hline \multicolumn{2}{|l|}{ Edmondson grade } \\
\hline I & $30.1 \%$ \\
\hline II & $50.4 \%$ \\
\hline III & $18.0 \%$ \\
\hline IV & $1.5 \%$ \\
\hline \multicolumn{2}{|l|}{ Grading } \\
\hline GI & $25.5 \%(58 / 227)$ \\
\hline G2 & $50.7 \%(115 / 227)$ \\
\hline G3 & $23.8 \%(54 / 227)$ \\
\hline \multicolumn{2}{|l|}{ Background liver parenchyma } \\
\hline Hepatitis & $39.9 \%(117 / 293)$ \\
\hline Cirrhosis & $28.3 \%(83 / 293)$ \\
\hline Normal liver parenchyma & $11.8 \%(37 / 3 \mid 3)$ \\
\hline Steatosis & $63.2 \%(36 / 57)$ \\
\hline Irregular histopathological structure & $97.6 \%(4 I / 42)$ \\
\hline Bulging of the tumor* & $100 \%(5 I / 5 I)$ \\
\hline Capsule & $26.3 \%(82 / 3 \mid 2)$ \\
\hline Septum & $85.6 \%(83 / 97)$ \\
\hline Central scar & $63.5 \%(40 / 63)$ \\
\hline Venous invasion & $32.6 \%(46 /|4|)$ \\
\hline \multicolumn{2}{|l|}{ Necrosis: } \\
\hline Present & $25.0 \%(58 / 232)$ \\
\hline Absent & $75.0 \%(174 / 232)$ \\
\hline
\end{tabular}

Note: *For tumors located near the capsule.

treatment and surveillance. The aim of this review was to summarize available knowledge on s-HCC and thus provide a solid basis for further research on s-HCCs.

The distribution of publications on s-HCC is striking. Although it is not surprising that countries with higher incidence of viral hepatitis, such as South Korea and Japan, would report on s-HCC more, the absence of publications from South America, Canada, China, Russia,

Table 4 Immunohistochemical Features of s-HCCs

\begin{tabular}{|l|c|}
\hline Immunohistochemical Feature & Prevalence \\
\hline HepParl positivity & $64.6 \%(64 / 99)$ \\
CK7 positivity & $40.7 \%(35 / 86)$ \\
CKI9 positivity & $16.0 \%(20 / 125)$ \\
EMA positivity & $41.9 \%(18 / 43)$ \\
\hline
\end{tabular}


Africa, and Europe is surprising and may be indicative of publication bias. Additionally, s-HCC has only recently been recognized as a separate subtype of $\mathrm{HCC}$, thus, some retrospective studies may perhaps still be pending.

Hepatitis B predominates in patients with s-HCC, afflicting approximately $60 \%$ of all reported cases, while hepatitis $\mathrm{C}$ causes liver disease in roughly an additional $20 \%$. These proportions roughly mirror previously reported viral causes of all HCC subtypes, ${ }^{48}$ although NAFLD and NASH have been rising as a cause of conventional HCC recently. ${ }^{49}$ NAFLD was not prevalent in s-HCC, neither was alcohol overindulgence. With different causes predominating in conventional versus scirrhous HCCs, the one-for-all therapy approach appears counterintuitive.

Individualized targeted therapy based on tumor receptor profiles has long been introduced in routine breast cancer treatment. ${ }^{50}$ System-based approach and genomic profiling have identified numerous potential targets in HCC treatment, such as Wnt/ $\beta$-catenin pathway, telomerases, and p53. ${ }^{51}$ However, an individualized therapy strategy is far from implementation for HCC patients. Additionally, no profiling has thus far been made of atypical HCCs, an approach with the potential to identify targets with adequate subtype and patient specificity.

S-HCC incorporates typical and atypical radiological and histopathological HCC features. While most $\mathrm{HCC}$ lesions appear round and hypoechoic on ultrasound, a mosaic pattern was just as frequent in s-HCC. Commonly, this feature appears in $\mathrm{HCC}$ with degenerative changes. ${ }^{52}$ Many imaging features of s-HCC seem to be related to its abundant desmoplastic tumor stroma. The fibrous stroma of s-HCC is presumably responsible for the frequent finding of retraction of the liver capsule, which is rare in untreated classical HCC. However, hepatic capsular retraction can occur in various other malignant and benign etiologies, including classical HCC with volume loss after locoregional therapy, other malignant liver tumors with fibrous stroma (cholangiocarcinoma, epithelioid hemangioendothelioma, FL-HCC), and confluent hepatic fibrosis, which often occurs in liver cirrhosis. ${ }^{53}$ Moreover, hepatic hemangiomas are more likely to become fibrotic and decrease in size in progressively cirrhotic livers and can retract the liver capsule. ${ }^{54}$ The dynamic contrast enhancement of s-HCC resembles that of cholangiocarcinomas, as both are rich in fibrous stroma. ${ }^{55}$ In contrast to the fast wash-in and wash-out of untreated conventional $\mathrm{HCC}$, s-HCC and cholangiocarcinomas frequently present with a peripheral rim APHE and progressive enhancement in the venous and delayed phases, whereby the areas of delayed enhancement often closely correspond to the presence of fibrotic fibers on histopathology. ${ }^{56}$ The presence of delayed enhancement in parts other than septae and the capsule in untreated HCC-lesions can be seen as indicative for a variant type of HCC, particularly s-HCC. ${ }^{56}$ However, delayed enhancement is also typical for metastases of colorectal adenocarcinoma. ${ }^{57}$

Although rare, the most frequent complications of biopsy are seeding and bleeding; thus, more emphasis has been placed on radiological imaging in the diagnostic algorithm. ${ }^{58}$ Yet, histopathological assessment is necessary for a definitive diagnosis. Most studies report experience with s-HCC after hepatectomy; however, since hepatitisassociated liver disease tends to precede s-HCC and many patients with $\mathrm{HCC}$ and liver cirrhosis can neither undergo surgery nor biopsy, a certain reporting bias must be assumed. ${ }^{58,59}$

Edmondson-Steiner classification is one of the most widely used histopathological classifications of $\mathrm{HCC}$. Most s-HCC patients had grade I/II with less than $25 \%$ presenting with grade III/IV, which contrasts general reports on $\mathrm{HCC} .^{60,61}$ It has been shown that ES grade may correlate with the recurrence of HCC. ${ }^{61}$ With $44.9 \%$ of the patients with available data presenting with a recurrence during follow-up and generally reported lower ES grades for s-HCC, this hypothesis is, although indirectly, supported.

Specific markers for distinct lesions are crucial. Since the identification of DNAJB1-PRKACA oncogenic fusion in FL-HCC that has high sensitivity, the accuracy of diagnosis improved, and novel therapeutic targets could be proposed. ${ }^{62}$ EMA is a marker of combined hepatocellular-cholangiocarcinoma ${ }^{63}$ and, interestingly, was positive in nearly half of all s-HCC cases $(41.9 \%)$ seeding doubt as to where s-HCC stands on the spectrum of HCC lesions. However, a sensitive marker for s-HCC is yet to be identified. Some authors suggest arginase-1 may be a more specific marker for HCC. ${ }^{64}$ This may also hold true for s-HCC; Krings et al described a cohort of 20 patients with s-HCC and 11 out of 13 cases were positive for arginase, while only 5 of 19 were positive for HepPar1. ${ }^{4}$ Unfortunately, this marker is widely unexplored in context of s-HCC. Additionally, polyclonal $\mathrm{CEA}^{65}$ may present an interesting stain for s-HCC, but thus far unexplored. Surprisingly, most s-HCCs were 
described to have a bulging appearance on histopathological examination when located near the capsule, yet, on radiological examination, surface retraction was most commonly reported - a clear contradiction, which warrants a comparative investigation.

The analyzed data showed a 5-year overall survival rate of $45.2 \%$, which is on the lower end of survival for HCC, as typical post-resection rates range from $41 \%$ to $74 \%{ }^{66}$ At the same time, the weight of the US registry significantly impacted the pooled mean, as it had the most patients and showed the lowest 5-year overall survival rates. Due to such discrepancies in reported survival rates, socioeconomic reasons and the structure of health care systems must be considered during the comparison. The recurrence rate reported was lower compared to the reported recurrence of up to $70 \%$ for all HCCs (68). With most patients presenting with hepatitis and cirrhosis and follow-up information lacking for most patients, it is vital to consider that the true recurrence and survival rates remain concealed and may lay far below currently presented.

\section{Certainty of Evidence}

The certainty of evidence within a systematic review or a meta-analysis is contingent on the design and methods of the included studies. It can be greatly affected by each publication and its risk of bias, as well as other factors such as inconsistency, imprecision, and indirectness. Additionally, a bias considerably affecting systematic reviews of rare conditions or interventions is publication bias. Inevitably, certainty in evidence derived from case series and reports will be very low. Nevertheless, inferences from these on rare and underreported conditions can be used for decision-making and is the best evidence on such afflictions to date.

The methodological quality assessment of included studies was performed using a tool proposed by Murad et al and assessed the domains of selection, ascertainment, causality, and reporting of the included case reports and case series. ${ }^{43}$ For observational studies, MINORS was additionally used as a risk of bias assessment. Overall, due to the study design and conceptual framework, the quality of evidence is very low.

\section{Conclusion}

S-HCC is a rare subtype of HCC that primarily arises in diseased livers with hepatitis or cirrhosis but lacking symptoms. S-HCC can often be misdiagnosed as fibrolamellar carcinoma, cholangiocarcinoma, or hepatic metastasis. Most cases of s-HCC have, perhaps, not been reported yet. Comparatively, low recurrence rate (45.5\%), yet poor 5-year survival rate $(45.2 \%)$ indicate that confounding factors may compromise patient life expectancy. A more comprehensive analysis of complex subtypes of HCC is possible with more reports from various parts of the world, and special emphasis must be placed on longterm outcomes after treatment, and clinical and histological features. Currently, patients with atypical lesions in the liver should be presented at tertiary referral hospitals for interdisciplinary assessment and treatment.

\section{Abbreviations}

AFP, alpha fetoprotein; AJCC, American Joint Committee on Cancer; APHE, arterial phase hyperenhancement; CK, cytokeratin; CT, computer tomography; EMA, epithelial membrane antigen; ES, Edmondson-Steiner grading; GRADE, Grading of Recommendations, Assessment, Development and Evaluations; HBP, hepatobiliary phase; HCC, hepatocellular carcinoma; HepPar1, hepatocyte paraffin 1 antigen; IPD, independent patient data; FL-HCC, fibrolamellar hepatocellular carcinoma; s-HCC, scirrhous hepatocellular carcinoma; MRI, magnetic; NASH, nonalcoholic steatohepatitis; RELIVE, initiative for generating evidence in diagnosis and therapy of RarE LIVEr disease; PRISMA, Preferred Reporting Items for Systematic Reviews and Meta-Analyses; WHO, World Health Organization.

\section{Data Sharing Statement}

All data generated or analyzed during this study are included in this published article and its Supplementary Materials.

\section{Author Contributions}

All authors contributed to data analysis, drafting or revising the article, gave final approval of the version to be published, agreed to the submitted journal, and agree to be accountable for all aspects of the work.

\section{Funding}

The authors have not declared a specific grant for this research from any funding agency in the public, commercial or not-for-profit sectors.

\section{Disclosure}

The authors declare that they have no conflicts of interest for this work. 


\section{References}

1. Nagtegaal ID, Odze RD, Klimstra D, et al. The 2019 WHO classification of tumours of the digestive system. Histopathology. 2020;76 (2):182-188. doi:10.1111/his.13975

2. Yang JD, Hainaut P, Gores GJ, Amadou A, Plymoth A, Roberts LR. A global view of hepatocellular carcinoma: trends, risk, prevention and management. Nat Rev Gastroenterol Hepatol. 2019;16 (10):589-604. doi:10.1038/s41575-019-0186-y

3. Torbenson MS. Morphologic subtypes of hepatocellular carcinoma. Gastroenterol Clin North Am. 2017;46(2):365-391. doi:10.1016/j. gtc.2017.01.009

4. Krings G, Ramachandran R, Jain D, et al. Immunohistochemical pitfalls and the importance of glypican 3 and arginase in the diagnosis of scirrhous hepatocellular carcinoma. Mod Pathol. 2013;26 (6):782-791. doi:10.1038/modpathol.2012.243

5. Kurogi M, Nakashima O, Miyaaki H, Fujimoto M, Kojiro M. Clinicopathological study of scirrhous hepatocellular carcinoma. J Gastroenterol Hepatol. 2006;21(9):1470-1477.

6. Limaiem F, Bouraoui S, Sboui M, Bouslama S, Lahmar A, Mzabi S. Fibrolamellar carcinoma versus scirrhous hepatocellular carcinoma: diagnostic usefulness of CD68. Acta Gastroenterol Belg. 2015;78 (4):393-398.

7. Min JH, Kim YK, Choi SY, et al. Differentiation between cholangiocarcinoma and hepatocellular carcinoma with target sign on diffusion-weighted imaging and hepatobiliary phase gadoxetic acid-enhanced MR imaging: classification tree analysis applying capsule and septum. Eur J Radiol. 2017;92:1-10. doi:10.1016/j. ejrad.2017.04.008

8. Lemekhova A, Hornuss D, Polychronidis G, et al. Clinical features and surgical outcomes of fibrolamellar hepatocellular carcinoma: retrospective analysis of a single-center experience. World $J$ Surg Oncol. 2020;18(1):93. doi:10.1186/s12957-020-01855-2

9. Vyas M, Hechtman JF, Zhang Y, et al. DNAJB1-PRKACA fusions occur in oncocytic pancreatic and biliary neoplasms and are not specific for fibrolamellar hepatocellular carcinoma. Mod Pathol. 2020;33(4):648-656. doi:10.1038/s41379-019-0398-2

10. Moher D, Liberati A, Tetzlaff J, Altman DG. Preferred reporting items for systematic reviews and meta-analyses: the PRISMA statement. PLoS Med. 2009;6(7):e1000097. doi:10.1371/journal. pmed.1000097

11. Lemekhova A, Fuchs J, Sterkenburg A, Hoffmann K. Scirrhous hepatocellular carcinoma: a systematic review of presentation and treatment outcomes. PROSPERO: International Prospective Register of Systematic Reviews; 2020.

12. Lefebvre CGJ, Briscoe S, Littlewood A, et al. Chapter 4: searching for and selecting studies. In: Higgins JPT, Thomas J, Chandler J, et al., editors. Cochrane Handbook for Systematic Reviews of Interventions Version 6.0 (Updated July 2019). Cochrane: 2019. Available from: www.training.cochrane.org/handbook. Accessed October 13, 2021.

13. R Core Team (2021). R: A language and environment for statistical computing. R Foundation for Statistical Computing, Vienna, Austria. Available from: https://www.R-project.org/.

14. Ariizumi S, Takasaki K, Otsubo T, Yamamoto M, Nakano M. Four immunohistochemically different primary liver cancers in one patient. J Gastroenterol. 2002;37(9):750-754. doi:10.1007/s005350200123

15. Chen F, Li H-J, Zhao D-W, Feng J-L, Ding J-L. Rare CT and MR imaging features of scirrhous hepatocellular carcinoma with gross specimen and pathologic correlation: case report and review of the literature. Radiol Infect Dis. 2015;2(3):137-140. doi:10.1016/j. jrid.2015.07.003

16. Choi SY, Kim YK, Min JH, et al. Added value of ancillary imaging features for differentiating scirrhous hepatocellular carcinoma from intrahepatic cholangiocarcinoma on gadoxetic acid-enhanced MR imaging. Eur Radiol. 2018;28(6):2549-2560. doi:10.1007/s00330017-5196-y
17. Chong YS, Kim YK, Lee MW, et al. Differentiating mass-forming intrahepatic cholangiocarcinoma from atypical hepatocellular carcinoma using gadoxetic acid-enhanced MRI. Clin Radiol. 2012;67 (8):766-773. doi:10.1016/j.crad.2012.01.004

18. Fujii T, Zen Y, Haradad K, et al. Participation of liver cancer stem/ progenitor cells in tumorigenesis of scirrhous hepatocellular carcinoma - human and cell culture study. Hum Pathol. 2008;39 (8):1185-1196. doi:10.1016/j.humpath.2007.12.010

19. Goshima S, Kanematsu M, Yamada T, et al. Small scirrhous hepatocellular carcinoma with central scar: MR imaging findings. $J$ Magn Reson Imaging. 2002;16(6):741-745. doi:10.1002/jmri.10211

20. Hatano M, Ojima H, Masugi Y, et al. Steatotic and nonsteatotic scirrhous hepatocellular carcinomas reveal distinct clinicopathological features. Hum Pathol. 2019;86:222-232. doi:10.1016/j. humpath.2018.11.024

21. Jakate S, Giusto D. Scirrhous hepatocellular carcinoma. Liver Pathol. 2011;4:417.

22. Jayasinghe B, Gill A, Irandoost P. A case report of a scirrhous variant of hepatocellular carcinoma (a rare variant). Pathology. 2014;46:S67. doi:10.1097/01.PAT.0000443561.20810.87

23. Jeon TY, Kim SH, Lee WJ, Lim HK. The value of gadobenate dimeglumine-enhanced hepatobiliary-phase MR imaging for the differentiation of scirrhous hepatocellular carcinoma and cholangiocarcinoma with or without hepatocellular carcinoma. Abdom Imaging. 2010;35(3):337-345. doi:10.1007/s00261-009-9509-8

24. Kim GJ, Rhee H, Yoo JE, et al. Increased expression of CCN2, epithelial membrane antigen, and fibroblast activation protein in hepatocellular carcinoma with fibrous stroma showing aggressive behavior. PLoS One. 2014;9(8):e105094. doi:10.1371/journal. pone. 0105094

25. Kim SH, Lim HK, Lee WJ, Choi D, Park CK. Scirrhous hepatocellular carcinoma: comparison with usual hepatocellular carcinoma based on CT-pathologic features and long-term results after curative resection. Eur $J$ Radiol. 2009;69(1):123-130. doi:10.1016/j. ejrad.2007.09.008

26. Kim SO, Baek HK, Shin BC, Jeong JS, Han SY, Lee SW. A case of brain metastasis from scirrhous type hepatocellular carcinoma. Korean J Med. 2009;77(5):1142-1147.

27. Kim SS, Hwang JA, Shin HC, et al. Synchronous occurrence of classic and scirrhous hepatocellular carcinomas: a case report. Iranian J Radiol. 2018;15:4.

28. Kim YJ, Rhee H, Yoo JE, et al. Tumour epithelial and stromal characteristics of hepatocellular carcinomas with abundant fibrous stroma: fibrolamellar versus scirrhous hepatocellular carcinoma. Histopathology. 2017;71(2):217-226. doi:10.1111/his.13219

29. Lee JH, Choi MS, Gwak GY, et al. Clinicopathologic characteristics and long-term prognosis of scirrhous hepatocellular carcinoma. Dig Dis Sci. 2012;57(6):1698-1707. doi:10.1007/ s10620-012-2075-x

30. Matsuura S, Aishima S, Taguchi K, et al. 'Scirrhous' type hepatocellular carcinomas: a special reference to expression of cytokeratin 7 and hepatocyte paraffin 1. Histopathology. 2005;47(4):382-390. doi:10.1111/j.1365-2559.2005.02230.x

31. Okamura N, Yoshida M, Shibuya A, Sugiura H, Okayasu I, Ohbu M. Cellular and stromal characteristics in the scirrhous hepatocellular carcinoma: comparison with hepatocellular carcinomas and intrahepatic cholangiocarcinomas. Pathol Int. 2005;55(11):724-731. doi:10.1111/j.1440-1827.2005.01891.x

32. Oshima G, Shinoda M, Tanabe M, et al. Primary hepatic cancers with multiple pathologic features in a patient with hepatitis C: report of a case. Int Surg. 2012;97(1):17-22. doi:10.9738/CC72.1

33. Park MJ, Kim YK, Park HJ, Hwang J, Lee WJ. Scirrhous hepatocellular carcinoma on gadoxetic acid-enhanced magnetic resonance imaging and diffusion-weighted imaging: emphasis on the differentiation of intrahepatic cholangiocarcinoma. J Comput Assist Tomogr. 2013;37(6):872-881. doi:10.1097/RCT.0b013e31829d44c1 
34. Sooklim K, Sriplung H, Piratvisuth T. Histologic subtypes of hepatocellular carcinoma in the southern Thai population. Asian Pac $J$ Cancer Prev. 2003;4(4):302-306.

35. Sugiki T, Yamamoto M, Aruga A, Takasaki K, Nakano M. Immunohistological evaluation of single small hepatocellular carcinoma with negative staining of monoclonal antibody Hepatocyte Paraffin 1. J Surg Oncol. 2004;88(2):104-107. doi:10.1002/jso.20144

36. Sugiki T, Yamamoto M, Taka K, Nakano M. Specific characteristics of scirrhous hepatocellular carcinoma. Hepatogastroenterology. 2009;56(93):1086-1089.

37. Tefera J, Revzin M, Chapiro J, et al. Fibronodular hepatocellular carcinoma-a new variant of liver cancer: clinical, pathological and radiological correlation. J Clin Pathol. 2020;74:31-35.

38. Yachida S, Wakabayashi H, Suzuki Y. Scirrhous hepatocellular carcinoma: unique computed tomography findings. Clin Gastroenterol Hepatol. 2009;7(1):A28. doi:10.1016/j.cgh.2008.06.017

39. Zakka K, Jiang RJ, Alese OB, et al. Clinical outcomes of rare hepatocellular carcinoma variants compared to pure hepatocellular carcinoma. J Hepatocell Carcinoma. 2019;6:119-129. doi:10.2147/ JHC.S215235

40. Masuda T, Mori T, Ikeda T, et al. A Case Report of Scirrhous Hepatocellular Carcinoma. The JapaneseJournal of Gastroenterological Surgery. 1991;24(3):876-879. Japanese. doi:10.5833/jjgs.24.876

41. Sadanobu I, Ohashi R, Onoda Y, Suzuka I, Shiota K. A Case of Scirrhous Hepatocellular Carcinoma with Various Histological Findings in Normal Liver. The Japanese Journal of Gastroenterological Surgery. 2006;39:1385-1390. Japanese. doi:10.5833/jjgs.39.1385

42. Huang SC, Liao SH, Su TH, Jeng YM, Kao JH. Clinical manifestations and outcomes of patients with scirrhous hepatocellular carcinoma. Hepatol Int. 2021;15(2):472-481. doi:10.1007/s12072021-10146-1

43. Murad MH, Sultan S, Haffar S, Bazerbachi F. Methodological quality and synthesis of case series and case reports. BMJ Evid Based Med. 2018;23(2):60-63. doi:10.1136/bmjebm-2017-110853

44. Slim K, Nini E, Forestier D, Kwiatkowski F, Panis Y, Chipponi J. Methodological index for non-randomized studies (minors): development and validation of a new instrument. ANZ J Surg. 2003;73 (9):712-716. doi:10.1046/j.1445-2197.2003.02748.x

45. Hollebecque A, Cattan S, Romano O, et al. Safety and efficacy of sorafenib in hepatocellular carcinoma: the impact of the Child-Pugh score. Aliment Pharmacol Ther. 2011;34(10):1193-1201. doi:10.1111/j.1365-2036.2011.04860.x

46. Facciorusso A, Tartaglia N, Villani R, et al. Lenvatinib versus sorafenib as first-line therapy of advanced hepatocellular carcinoma: a systematic review and meta-analysis. Am J Transl Res. 2021;13 (4):2379-2387.

47. Chan EE, Chow PK. A review of prognostic scores after liver resection in hepatocellular carcinoma: the MSKCC, SLICER and SSCLIP scores. Jpn J Clin Oncol. 2017;47(4):287-293.

48. Ghouri YA, Mian I, Rowe JH. Review of hepatocellular carcinoma: epidemiology, etiology, and carcinogenesis. J Carcinog. 2017;16:1. doi:10.4103/jcar.JCar 916

49. Sayiner M, Golabi P, Younossi ZM. Disease burden of hepatocellular carcinoma: a global perspective. Dig Dis Sci. 2019;64(4):910-917. doi:10.1007/s10620-019-05537-2

50. Masoud V, Pagès G. Targeted therapies in breast cancer: new challenges to fight against resistance. World J Clin Oncol. 2017;8 (2):120-134. doi:10.5306/wjco.v8.i2.120
51. Ozcan M, Altay O, Lam S, et al. Improvement in the current therapies for hepatocellular carcinoma using a systems medicine approach. Adv Biosyst. 2020;4(6):e2000030. doi:10.1002/adbi.202000030

52. Nowicki TK, Markiet K, Szurowska E. Diagnostic imaging of hepatocellular carcinoma - a pictorial essay. Curr Med Imaging Rev. 2017;13(2):140-153. doi:10.2174/1573405612666160720123748

53. Blachar A, Federle MP, Brancatelli G. Hepatic capsular retraction: spectrum of benign and malignant etiologies. Abdom Imaging. 2002;27(6):690-699. doi:10.1007/s00261-001-0094-8

54. Brancatelli G, Federle MP, Blachar A, Grazioli L. Hemangioma in the cirrhotic liver: diagnosis and natural history. Radiology. 2001;219 (1):69-74. doi:10.1148/radiology.219.1.r01ap3269

55. Gentilini A, Pastore M, Marra F, Raggi C. The role of stroma in cholangiocarcinoma: the intriguing interplay between fibroblastic component, immune cell subsets and tumor epithelium. Int $J \mathrm{Mol}$ Sci. 2018;19(10):2885. doi:10.3390/ijms19102885

56. Yoshikawa J, Matsui O, Kadoya M, Gabata T, Arai K, Takashima T. Delayed enhancement of fibrotic areas in hepatic masses: CT-pathologic correlation. J Comput Assist Tomogr. 1992;16:2. doi:10.1097/ 00004728-199203000-00006

57. Cheung HMC, Karanicolas PJ, Coburn N, Law C, Milot L. Tumor enhancement of colorectal liver metastases on preoperative gadobutrol-enhanced MRI at 5 minutes post-contrast injection is associated with overall survival post-hepatectomy. Quant Imaging Med Surg. 2019;9(2):312-317. doi:10.21037/qims.2018.10.15

58. Russo FP, Imondi A, Lynch EN, Farinati F. When and how should we perform a biopsy for HCC in patients with liver cirrhosis in 2018? A review. Dig Liver Dis. 2018;50(7):640-646. doi:10.1016/j. dld.2018.03.014

59. Llovet JM, Fuster J, Bruix J. The Barcelona approach: diagnosis, staging, and treatment of hepatocellular carcinoma. Liver Transpl. 2004;10(2 Suppl 1):S115-20. doi:10.1002/lt.20034

60. Tunissiolli NM, Castanhole-Nunes MMU, Pavarino ÉC, et al. Clinical, epidemiological and histopathological aspects in patients with hepatocellular carcinoma undergoing liver transplantation. Asian Pac $j$ Cancer Prev. 2018;19(10):2795-2802.

61. Zhou L, Wang SB, Chen SG, Qu Q, Rui JA. Risk factors of recurrence and poor survival in curatively resected hepatocellular carcinoma with microvascular invasion. Adv Clin Exp Med. 2020;29 (7):887-892. doi:10.17219/acem/76750

62. Lalazar G, Simon SM. Fibrolamellar carcinoma: recent advances and unresolved questions on the molecular mechanisms. Semin Liver Dis. 2018;38(1):51-59. doi:10.1055/s-0037-1621710

63. Schlageter M, Terracciano LM, D'Angelo S, Sorrentino P. Histopathology of hepatocellular carcinoma. World J Gastroenterol. 2014;20(43):15955-15964. doi:10.3748/wjg.v20.i43.15955

64. Radwan NA, Ahmed NS. The diagnostic value of arginase-1 immunostaining in differentiating hepatocellular carcinoma from metastatic carcinoma and cholangiocarcinoma as compared to HepPar-1. Diagn Pathol. 2012;7(1):149. doi:10.1186/1746-1596-7-149

65. Morrison C, Marsh W Jr, Frankel WL. A comparison of CD10 to pCEA, MOC-31, and hepatocyte for the distinction of malignant tumors in the liver. Mod Pathol. 2002;15(12):1279-1287. doi:10.1097/01.MP.0000037312.69565.24

66. Balogh J, Victor D 3rd, Asham EH, et al. Hepatocellular carcinoma: a review. J Hepatocell Carcinoma. 2016;3:41-53. doi:10.2147/JHC. S61146 


\section{Publish your work in this journal}

The Journal of Hepatocellular Carcinoma is an international, peerreviewed, open access journal that offers a platform for the dissemination and study of clinical, translational and basic research findings in this rapidly developing field. Development in areas including, but not limited to, epidemiology, vaccination, hepatitis therapy, pathology

and molecular tumor classification and prognostication are all considered for publication. The manuscript management system is completely online and includes a very quick and fair peer-review system, which is all easy to use. Visit http://www.dovepress.com/ testimonials.php to read real quotes from published authors.

Submit your manuscript here: https://www.dovepress.com/journal-of-hepatocellular-carcinoma-journal 